\title{
Emergency Medical Services Systems
}

\author{
Marvin L. Birnbaum, MD, PhD
}

\author{
It is folly to expect men to do all \\ That they may reasonably be expected to do. \\ Richard Whatley, Apothegms \\ Blessed be he who expects nothing, \\ For be shall never be disappointed. \\ John Wolcott, Ode to Pitt, 1,1
}

Overall, our view of Emergency Medical Services (EMS) is too constrained and limits services that could be provided. Some time ago, a friend remarked that EMS was unrelated to Disaster Medicine and, in fact, advised that the World Association for Disaster and Emergency Medicine (WADEM) and this journal (PDM) needed to concentrate either on Disaster Medicine $\underline{O R}$ EMS. More recently, this same friend came to understand that Disaster Medicine could not work effectively and efficiently without the services provided by an EMS System. The key word was "system".

A system is defined as: a regularly interacting or interdependent group of items forming a unified whole. ${ }^{1}$ The question is: What constitutes the "whole?" The late Dr. Peter Safar, in his pioneering work with the development of the Pittsburgh EMS System, envisioned the system as a continuum beginning with entry into the system until discharge. But, today, the "whole" is even broader than initially conceived by Dr. Safar. The system now encompasses public health, raising awareness, needs assessments, emergent clinic visits, communicators and communications centers, education and training, hospital receiving facilities, and primary health care.

Unfortunately, as my friend initially believed, almost all of the established healthcare systems have come to view EMS as the prehospital emergency response to, assessment of, treatment of, and transport of someone who calls for medical assistance. This is a very narrow perspective: the broad sweep that should encompass EMS has been lost, and EMS systems worldwide have become fragmented and isolated. The systems have been deconstructed into their various components. This isolated, prehospital perception of Emergency Medical Services undoubtedly biased my friend's perception of the role of EMS in the world of Emergency and Disaster Medicine. Furthermore, in many countries and in particular in the US, the same deconstruction of EMS systems has been happening to the other components of the EMS system, e.g., emergency medicine, emergency nursing, and critical medicine and nursing, and especially public health and primary health care.

Consequently, it is difficult to comprehend the broad scope of EMS Systems. Given this narrow view, it has been difficult to obtain the resources necessary to fund EMS systems. Generally, funding has been made available only for those services in the system that have high visibility. Other less visible components have been under-appreciated and continue to be under-funded. A lack of adequate support further spurs the parochialism that has resulted from fragmentation. Components and their respective processes now fight for their own individual existence. We have lost the system concept.

This dissection of the "system" into a few of its components has spread into the scope of the education and training provided. These educational and training programs now limit the scope of what is being taught. The educational goals are confined with little added appreciation of the whole system. The committed personnel that comprise the system constitute a huge, under-utilized resource. Furthermore, such practices interfere with the professionalization of the broader discipline.

There are many benefits that can be accrued by broadening the scope of what is taught and how it is practiced. The scope of what is taught and provided should encompass those services that meet more of the specific and potential needs of the communities they serve. The result would be the provision of services that currently are not being provided or for which access is limited. Such practices should better meet the public health needs of the society, including an enhanced level of preparedness to cope with all of the hazards for which the society is at risk. Emergency Medical Services systems should provide the front line of defense through the provision of surveillance, immunizations, enhancing awareness, as well as response once the event occurs. As such, they should be an important component of public health.

This short-sightedness also has fostered "research" that reflects the narrowness of scope. Many of the published works have been so narrow as to ignore many of the effects of the intervention being studied on other components of the EMS system. Very little EMS system research has been published, probably because knowledge of the system was not appreciated. Few studies have attempted to compare different EMS systems especially in relation to culture and needs of the respective societies in which they operate. Which systems work better and why? What are the specific needs of the society served? Further, there is a paucity of outcome data: we know little about the effectiveness, efficacy, efficiency, costs, and benefits of interventions or of the services for which the studies were accomplished. It is important that researchers broaden their discussions and to examine the more far-reaching aspects of what they have done.

Little is known about the mandatory roles of EMS systems in the provision of some of the elements of primary 
care in societies in which EMS may provide the best answer to some of these needs. Enhancing awareness of the system in the community, its components, and how it works has not been emphasized. Unfortunately, the expectations of the population largely have been established through the non-medical media. The potential roles of EMS Systems in public health have been neglected by almost all of its other components.

We have allowed the deconstruction of the "system" for convenience and because of lack of resources. Deconstruction has its place in understanding how the system works and in identifying components that do not work. Emergency Medical Services Systems are a vital part of meeting the needs of the society they serve including disaster preparedness, response, public health, and primary care. When designing systems for the developing parts of the globe, careful attention must be given to the needs of the society as well as the availability of resources. A comprehensive EMS system can provide many services not traditionally thought of as part of EMS even in Services that are welldeveloped. Existing services should examine the possibilities for expanding their services they provide to their constituents. It is in this way that the single discipline of emergency and disaster medicine, and public health truly can fulfill its potential.

Courage is that virtue which champions the cause of the right. (Fortitude eam virtutem propugnantem pro aequitate.)

Richard Whatley, Apothegms

Why now I see there's mettle in thee.

Shakespeare, Otbello, Act iv, sc 2

Reference

1. Mish FC (ed): Merriam-Webster's Collegiate Dictionary, 10th ed, Merriam Webster, Inc.: Springfield, Massachusetts, USA. p 1197. 